\title{
Subject Index Vol. 12, 1996
}

Aberration currents S2 13

Acinar cells SI 105

Acute necrotizing pankreatitis 4358

- pancreatitis 166

Adult polycystic liver disease: surgical

treatment, reresection 2146 Application (monopolar, bipolar) S2 13 Ascites 4299 -,

(therapy) 4326

B-Adrenoreceptor blockers 4295 Bile duct injury S2 17

ligation 2153

stones 132

Biliary endoprosthesis 115 Bladder drainage technique SI 16 -, future perspectives SI 16

Blood flow 4283 Bougie 16

Capnoperitoneum S2 10

Carcinogenesis 3232

Chronic pancreatitis $138,3222,3232$

- $\quad$-, diagnosis, US, CT, ERCP, clinical

score 3192

cysts, operative therapy 3228

indications for surgery, surgical options, results 3214

, pancreatic insufficiency (exocrine,

endocrine), pain, alcohol 3188

, pancreatic insufficiency, pancreatic

pain, enzyme replacement therapy, endoscopic interventional therapy, extracorporeal shock-

wave lithot-ripsy (ESWL) 3206

, pathomorphology, surgical

therapy 3198

Cirrhosis of the liver 4278

Color flow doppler sonography SI 54

Combined pankreas/kidney transplantation SI 84

- $\quad$-, quality of life SI 84 , telephone interview SI 84

Computed tomotgraphy SI 54

Crohn's disease: operative therapy 2126

Current density S2 13

Day and short stay surgery: efficiency, nursing 143

Devascularization procedure 4314

Diabetes mellitus SI 73

-, polyneuropathies SI 73

-, autonomic neuropathies SI 73 
-, remission type SI 73

Diuretics 4326

Duodenum-preserving resection of the pancreatic head (morbidity, mortality)

3222

Efficacy of nutritional therapy 2108

Encephalopathy 4299

Endoscopic therapy 1 6, 132

Endoscopic treatment of festul as 115

Endoscopy 120

Endothelins 4278

Enteral nutrition 2112

-, postoperative 2104

ERCP 132

Esophageal and gastric varices 4289

- $\quad$ varices 4 283, 4 308ESWL 138

Fibrin glue 115 Fluoroscopy 120 Fundoplication S2 30

Gallblader tumour 3250 Gastroenterostomy S2 35 Gastroesophageal reflux S2 30 disease: Nissen fundoplication,

hiatal repair, laparoscopic technique, results of treatment S2 41 Gastrointestinal festulas 115

hemorrhage 4289

tract 16

tumors S2 35 Gastrostomy S2 35 -, method 120 Glucose 2119

metabolism 2119

tolerance SI 63 Graft pancreatitis SI 44 -, acute SI 105

-, chronic SI 105 -, thrombosis SI 44 -, ultrastructure SI 105

Hemobilia: etiology, surgical treatment

2132

Hepatic encephalopathy 290 -, therapy, grading 4338

- $\quad$ failure 290 Hiatal hernia S2 30High-frequency current S2 13 Hyperdynamic systemic circulation 4 278Hyperinsulinemia SI 63Hypertriglyceridemia $166 \mathrm{Hypocaloric}$ parenteral nutrition 2108

Immunonutrition 2104 Immunosuppression SI 38, SI 63 Inguinal hernia 171 Insulin resistance SI 63 Interleukin-2 2153 Interleukin-3 2153 Internal biliary drainage 2153

Intervention 16 Interventional radiology 4299 Intraoperative cholangiography S2 17 IPTR SI 88 Islet cells SI 105

Laparoscopic appendectomy (laparoscopic vs. open approach, operative technique, wound infection risk) S2 25

cholecystectomy S2 17

hernia repair 171

- $\quad$ instruments: instrument care, sterilization S2 6

Laparoscopy 120, S2 10, S2 30 -. contraindications, documentation, informed consent of patients S2 1 Leakage current S2 13 Limited currentflow S2 13 Liver 4299

cirrhosis $4295,4299,4314$

disease 3250

function 4344 
perfusion 4344

resection 3250

transplantation 4321

tumour 3250

Magnetic resonance imaging SI 54 Malign bile duct stenosis 132

Nitric oxide 4278

Obstructive jaundice 2153

\section{KARGER}

(C) 1996 S. KargerGmbH, Freiburg Fax (0761)45207 14

hUp://www.karger.ch/journals/cga/cgades.ritrrı

Papillotomy 132

Palliation S2 35

Palliative endoscopic pertubation 115

Pancreas retrieval SI 32

-, vascular anatomy SI 32

-, arterial reconstruction SI 32

Pancreas transplantation SI 9, SI 16,

SI 38 , SI 44, SI 54, SI 73, SI 88 ,

SI 98 , SI 111

, complications SI 44

, immunosuppression SI 94

, indication SI 9

, long-term follow-up SI 98

, recipient selection SI 9

- $\quad$ - $\quad$, results SI 94

, surgical technique SI 16 , SI 98

Pancreatitis SI 111

-, definition, classification, pathogene-

sis, clinical significance 3182 -, pathophysiology SI 111 Pancreas/Kidney transplantation SI 23 ,

SI 50

, anesthesia SI 23

, infection SI 50

, pathophysiology SI 23

: pancreatic pseudocysts, surgical

and interventional treatment 126

, rejection SI 50

, survival analysis SI 50

Pancreatic carcinoma 3232

innervation SI 63

transplantation, diabetic complications SI 76

Pancreaticoduodenal/kidney transplantation SI 102

- $\quad$-, results SI 102Paracentesis 4326

Parenteral nutrition 2 100,2 112,2 119 Peritoneovenous shunt 4326 Peritonitis (fecal, severe) 4358 -, animal model, Taurolin ${ }^{\circledR}-/ \mathrm{NaCl}$-lavage 
3 253Pneumoperitoneum S2 10

Portal hypertension 4 278, 4 283, 4 299,

$4 \quad 321$

Portal hypotension 4344 Portal vein 4299 Portosystemic shunt 4321 Postoperative

complications 132

metabolism 2100

nitrogen balance 2108

nutrition 290

nutritional support 2112

Rectal cancer: randomised study protocols, concomitant radio-/chemothera-py, pre-operative Radiotherapy 2136

Recurrent bleeding from esophagogastric varices 4314

- $\quad$ inguinal hernia 171Rejection SI 38

-, therapy SI 38

Renal failure (acute, chronic) 2112

Rubber band ligation 4308

Scintigraphy SI 54

Sclerotherapy 4308

Sepsis 2 100,2 104,2 119

Shunt procedure 4314

-, portosystemic 4299

Small hepatocellular carcinoma: surgery,

therapy, prognosis 3244 Somatostatin 4295 Sphincterotomy 138 Spontaneous bacterial peritonitis 4326 Stable isotopes 2119 Stenoses 16 Stenting 138 Stomata S2 35 Substrate metabolism 290

Taurolidin 4358

Transabdominal preperitoneal patch plastic 171 Transjugular portosystemic shunt 4326

Trauma 2119 Type 1 diabetes SI 63

Upper gastrointestinal bleeding: endoscopic hemostasis, fibrin sealant, recurrent bleeding 1 43

Variceal bleeding 4 295,4 321 Vascular resistance 4283 Vasopressin 4295

Xylitol 2119

372

Chir Gastroenterol 1996; 12

Subject Index Vol. 12, 1996 